\title{
Arbitrary decays for a viscoelastic equation
}

\author{
Shun-Tang Wu
}

Correspondence: stwu@ntut.edu.tw General Education Center National Taipei University of Technology Taipei 106, Taiwan

\begin{abstract}
In this paper, we consider the nonlinear viscoelastic equation

$\left|u_{t}\right|^{\rho} u_{t t}-\Delta u-\Delta u_{t t}+\int_{0}^{t} g(t-s) \Delta u(s) \mathrm{d} s+|u|^{p} u=0$, in a bounded domain with initial conditions and Dirichlet boundary conditions. We prove an arbitrary decay result for a class of kernel function $g$ without setting the function $g$ itself to be of exponential (polynomial) type, which is a necessary condition for the exponential (polynomial) decay of the solution energy for the viscoelastic problem. The key ingredient in the proof is based on the idea of Pata (Q Appl Math 64:499-513, 2006) and the work of Tatar (J Math Phys 52:013502, 2010), with necessary modification imposed by our problem.
\end{abstract}

Mathematical Subject Classification (2010): 35B35, 35B40, 35B60

Keywords: Viscoelastic equation, Kernel function, Exponential decay, Polynomial decay

\section{Introduction}

It is well known that viscoelastic materials have memory effects. These properties are due to the mechanical response influenced by the history of the materials themselves. As these materials have a wide application in the natural sciences, their dynamics are of great importance and interest. From the mathematical point of view, their memory effects are modeled by an integro-differential equations. Hence, questions related to the behavior of the solutions for the PDE system have attracted considerable attention in recent years. Many authors have focused on this problem for the last two decades and several results concerning existence, decay and blow-up have been obtained, see [1-28] and the reference therein.

In [3], Cavalcanti et al. studied the following problem

$$
\begin{aligned}
& \left|u_{t}\right|^{\rho} u_{t t}-\Delta u-\Delta u_{t t}+\int_{0}^{t} g(t-s) \Delta u(s) \mathrm{d} s-\gamma \Delta u_{t}=0, \quad \text { in } \Omega \times(0, \infty), \\
& u(x, 0)=u_{0}(x), u_{t}(x, 0)=u_{1}(x), x \in \Omega, \\
& u(x, t)=0, x \in \partial \Omega, t \geq 0,
\end{aligned}
$$

where $\Omega \subset R^{N}, N \geq 1$, is a bounded domain with a smooth boundary $\partial \Omega, \gamma \geq 0$, $0<\rho \leq \frac{2}{N-2}$ if $N \geq 3$ or $\rho>0$ if $N=1,2$, and the function $g: R^{+} \rightarrow R^{+}$is a nonincreasing function. This type of equations usually arise in the theory of viscoelasticity when the material density varies according to the velocity. In that paper, they proved a global existence result of weak solutions for $\gamma \geq 0$ and a uniform decay result for $\gamma>0$.

(c) 2011 Wu; licensee Springer. This is an Open Access article distributed under the terms of the Creative Commons Attribution License (http://creativecommons.org/licenses/by/2.0), which permits unrestricted use, distribution, and reproduction in any medium, provided the original work is properly cited. 
Precisely, they showed that the solutions goes to zero in an exponential rate for $\gamma>0$ and $g$ is a positive bounded $C^{1}$-function satisfying

$$
1-\int_{0}^{\infty} g(s) \mathrm{d} s=1-l>0
$$

and

$$
-\xi_{1} g(t) \leq g^{\prime}(t) \leq-\xi_{2} g(t),
$$

for all $t \geq 0$ and some positive constants $\xi_{1}$ and $\xi_{2}$. Later, this result was extended by Messaoudi and Tatar [15] to a situation where a nonlinear source term is competing with the dissipation terms induced by both the viscoelasticity and the viscosity. Recently Messaoudi and Tatar [14] studied problem (1.1) for the case of $\gamma=0$, they improved the result in [3] by showing that the solution goes to zero with an exponential or polynomial rate, depending on the decay rate of the relaxation function $g$.

The assumptions (1.2) and (1.3), on $g$, are frequently encountered in the linear case $(\rho=0)$, see $[1,2,4-6,13,22,23,29-31]$. Lately, these conditions have been weakened by some researchers. For instance, instead of (1.3) Furati and Tatar [8] required the functions $\mathrm{e}^{\alpha t} g(t)$ and $\mathrm{e}^{\alpha t} g^{\prime}(t)$ to have sufficiently small $L^{1}$-norm on $(0, \infty)$ for some $\alpha>0$ and they can also have an exponential decay of solutions. In particular, they do not impose a rate of decreasingness for $g$. Later on Messaoudi and Tatar [21] improved this result further by removing the condition on $g$ '. They established an exponential decay under the conditions $g^{\prime}(t) \leq 0$ and $\mathrm{e}^{\alpha t} g(t) \in L^{1}(0, \infty)$ for some large $\alpha>0$. This last condition was shown to be necessary condition for exponential decay [7]. More recently Tatar [25] investigated the asymptotic behavior to problem (1.1) with $\rho=\gamma=$ 0 when $h(t) g(t) \in L^{1}(0, \infty)$ for some nonnegative function $h(t)$. He generalized earlier works to an arbitrary decay not necessary of exponential or polynomial rate.

Motivated by previous works $[21,25]$, in this paper, we consider the initial boundary value problem for the following nonlinear viscoelastic equation:

$$
\left|u_{t}\right|^{\rho} u_{t t}-\Delta u-\Delta u_{t t}+\int_{0}^{t} g(t-s) \Delta u(s) \mathrm{d} s+|u|^{p} u=0, \quad \text { in } \Omega \times(0, \infty),
$$

with initial conditions

$$
u(x, 0)=u_{0}(x), u_{t}(x, 0)=u_{1}(x), x \in \Omega,
$$

and boundary condition

$$
u(x, t)=0, x \in \partial \Omega, t \geq 0,
$$

where $\Omega \subset R^{N}, N \geq 1$, is a bounded domain with a smooth boundary $\partial \Omega$. Here $\rho$, $p>0$ and $g$ represents the kernel of the memory term, with conditions to be stated later [see assumption (A1)-(A3)].

We intend to study the arbitrary decay result for problem (1.4)-(1.6) under the weaker assumption on $g$, which is not necessarily decaying in an exponential or polynomial fashion. Indeed, our result will be established under the conditions $g^{\prime}(t) \leq 0$ and $\int_{0}^{\infty} \xi(s) g(s) \mathrm{d} s<\infty$ for some nonnegative function $\xi(t)$. Therefore, our result allows a 
larger class of relaxation functions and improves some earlier results concerning the exponential decay or polynomial decay.

The content of this paper is organized as follows. In Section 2, we give some lemmas and assumptions which will be used later, and we mention the local existence result in Theorem 2.2. In Section 3, we establish the statement and proof of our result related to the arbitrary decay.

\section{Preliminary results}

In this section, we give some assumptions and lemmas which will be used throughout this work. We use the standard Lebesgue space $L^{p}(\Omega)$ and Sobolev space $H_{0}^{1}(\Omega)$ with their usual inner products and norms.

Lemma 2.1. (Sobolev-Poincaré inequality) Let $2 \leq p \leq \frac{2 N}{N-2}$, the inequality

$$
\|u\|_{p} \leq c_{s}\|\nabla u\|_{2} \quad \text { for } u \in H_{0}^{1}(\Omega),
$$

holds with the optimal positive constant $c_{s}$, where $\|\cdot\|_{p}$ denotes the norm of $L^{p}(\Omega)$. Assume that $\rho$ satisfies

$$
0<\rho \leq \frac{2}{N-2} \text { if } N \geq 3 \text { or } \rho>0 \quad \text { if } N=1,2
$$

With regards to the relaxation function $g(t)$, we assume that it verifies

(A1) $g(t) \geq 0$, for all $t \geq 0$, is a continuous function satisfying

$$
0<\int_{0}^{\infty} g(s) \mathrm{d} s=l<1 .
$$

(A2) $g^{\prime}(t) \leq 0$ for almost all $t>0$.

(A3) There exists a positive nondecreasing function $\xi(t):[0, \infty) \rightarrow(0, \infty)$ such that $\frac{\xi^{\prime}(t)}{\xi(t)}=\eta(t)$ is a decreasing function and

$$
\int_{0}^{\infty} \xi(s) g(s) \mathrm{d} s<\infty .
$$

Now, we state, without a proof, the existence result of the problem (1.4)-(1.6) which can be established by Faedo-Galerkin methods, we refer the reader to $[3,5]$.

Theorem 2.2. Suppose that (2.1) and (A1) hold, and that $\left(u_{0}, u_{1}\right) \in H_{0}^{1}(\Omega) \times H_{0}^{1}(\Omega)$. Assume $0<\rho \leq \frac{2}{N-2}$, if $N \geq 3, p>0$, if $N=1,2$. Then there exists at least one global solution $u$ of (1.4)-(1.6) satisfying

$$
u \in L^{\infty}\left([0, \infty) ; H_{0}^{1}(\Omega)\right), u_{t} \in L^{\infty}\left([0, \infty) ; H_{0}^{1}(\Omega)\right), u_{t t} \in L^{2}\left([0, \infty) ; L^{2}(\Omega)\right) .
$$

Next, we introduce the modified energy functional for problem (1.4)-(1.6)

$$
\begin{aligned}
E(t) & =\frac{1}{\rho+2}\left\|u_{t}\right\|_{\rho+2}^{\rho+2}+\frac{1}{2}\left(1-\int_{0}^{t} g(s) \mathrm{d} s\right)\|\nabla u(t)\|_{2}^{2}+\frac{1}{2}\left\|\nabla u_{t}(t)\right\|_{2}^{2} \\
& +\frac{1}{2}(g \circ \nabla u)(t)+\frac{1}{p+2}\|u(t)\|_{p+2}^{p+2}
\end{aligned}
$$


where

$$
(g \circ \nabla u)(t)=\int_{0}^{t} \int_{\Omega} g(t-s)|\nabla u(t)-\nabla u(s)|^{2} \mathrm{~d} x \mathrm{~d} s .
$$

Lemma 2.3. Let $u$ be the solution of (1.4)-(1.6), then the modified energy $E(t)$ satisfies

$$
E^{\prime}(t)=\frac{1}{2}\left(g^{\prime} \circ \nabla u\right)(t)-\frac{1}{2} g(t)\|\nabla u(t)\|_{2}^{2} \leq \frac{1}{2}\left(g^{\prime} \circ \nabla u\right)(t) \leq 0 .
$$

Proof. Multiplying Eq. (1.4) by $u_{t}$ and integrating it over $\Omega$, then using integration by parts and the assumption (A1)-(A2), we obtain (2.6).

Remark. It follows from Lemma 2.3 that the energy is uniformly bounded by $E(0)$ and decreasing in $t$. Besides, from the definition of $E(t)$ and $(2,2)$, we note that

$$
(1-l)\|\nabla u\|_{2}^{2}+\left\|\nabla u_{t}(t)\right\|_{2}^{2}+(g \circ \nabla u)(t) \leq 2 E(0), \quad \forall t \geq 0 .
$$

\section{Decay of the solution energy}

In this section, we shall state and prove our main result. For this purpose, we first define the functional

$$
L(t)=E(t)+\sum_{i=1}^{3} \lambda_{i} \Phi_{i}(t)
$$

where $\lambda_{i}$ are positive constants, $i=1,2,3$ to be specified later and

$$
\begin{aligned}
& \Phi_{1}(t)=\frac{1}{\rho+1} \int_{\Omega}\left|u_{t}\right|^{\rho} u_{t} u \mathrm{~d} x+\int_{\Omega} \nabla u_{t}(t) \nabla u(t) \mathrm{d} x, \\
& \Phi_{2}(t)=\int_{\Omega}\left(\Delta u_{t}-\frac{1}{\rho+1}\left|u_{t}\right|^{\rho} u_{t}\right) \int_{0}^{t} g(t-s)(u(t)-u(s)) \mathrm{d} s \mathrm{~d} x, \\
& \Phi_{3}(t)=\int_{\Omega} \int_{0}^{t} H(t-s)|\nabla u(s)|^{2} \mathrm{~d} s \mathrm{~d} x,
\end{aligned}
$$

here

$$
H(t)=\xi(t)^{-1} \int_{t}^{\infty} g(s) \xi(s) \mathrm{d} s .
$$

Remark. This functional was first introduced by Tatar [25] for the case of $\rho=0$ and without imposing the dispersion term and forcing term as far as (1.4) is concerned.

The following Lemma tells us that $L(t)$ and $E(t)+\Phi_{3}(t)$ are equivalent. 
Lemma 3.1. There exists two positive constants $\beta_{1}$ and $\beta_{2}$ such that the relation

$$
\beta_{1}\left(E(t)+\Phi_{3}(t)\right) \leq L(t) \leq \beta_{2}\left(E(t)+\Phi_{3}(t)\right)
$$

holds for all $t \geq 0$ and $\lambda_{i}$ small, $i=1,2$.

Proof. By Hölder inequality Young's inequality Lemma 2.1, (2.7) and (2.2), we deduce that

$$
\begin{aligned}
&\left.\left|\int_{\Omega}\right| u_{t}\right|^{\rho} u_{t} u \mathrm{~d} x \mid \leq \frac{1}{2}\left\|u_{t}\right\|_{2(\rho+1)}^{2(\rho+1)}+\frac{1}{2}\|u\|_{2}^{2} \\
& \leq \frac{c_{s}^{2(\rho+1)}}{2}\left\|\nabla u_{t}\right\|_{2}^{2(\rho+1)}+\frac{c_{s}^{2}}{2}\|\nabla u\|_{2}^{2} \\
& \leq \frac{\alpha_{1}}{2}\left\|\nabla u_{t}\right\|_{2}^{2}+\frac{c_{s}^{2}}{2}\|\nabla u\|_{2}^{2} \\
&\left|\int_{\Omega} \nabla u_{t}(t) \nabla u(t) \mathrm{d} x\right| \leq \frac{1}{2}\left(\left\|\nabla u_{t}\right\|_{2}^{2}+\|\nabla u\|_{2}^{2}\right), \\
&\left|\int_{\Omega} \nabla u_{t} \int_{0}^{t} g(t-s)(\nabla u(t)-\nabla u(s)) \mathrm{d} s \mathrm{~d} x\right| \\
& \leq \frac{1}{2}\left\|\nabla u_{t}\right\|_{2}^{2}+\frac{1}{2} \int_{\Omega}\left(\int_{0}^{t} g(t-s)(\nabla u(t)-\nabla u(s))\right)^{2} \mathrm{~d} s \mathrm{~d} x \\
& \leq \frac{1}{2}\left\|\nabla u_{t}\right\|_{2}^{2}+\frac{l}{2}(g \circ \nabla u)(t),
\end{aligned}
$$

and

$$
\begin{aligned}
& \left.\left|\int_{\Omega}\right| u_{t}\right|^{\rho} u_{t} \int_{0}^{t} g(t-s)(u(t)-u(s)) \mathrm{d} s \mathrm{~d} x \mid \\
& \quad \leq \frac{1}{2}\left\|u_{t}\right\|_{2(\rho+1)}^{2(\rho+1)}+\frac{1}{2} \int_{\Omega}\left(\int_{0}^{t} g(t-s)(u(t)-u(s))\right)^{2} \mathrm{~d} s \mathrm{~d} x \\
& \quad \leq \frac{\alpha_{1}}{2}\left\|\nabla u_{t}\right\|_{2}^{2}+\frac{l c_{s}^{2}}{2}(g \circ \nabla u)(t),
\end{aligned}
$$

where $\alpha_{1}=c_{s}^{2(\rho+1)}(2 E(0))^{\rho}$. Therefore, from above estimates, the definition of $E(t)$ by (2.4) and (2.2), we have

$$
\begin{aligned}
L(t) & =E(t)+\sum_{i=1}^{3} \lambda_{i} \Phi_{i}(t) \\
& \leq E(t)+c_{1}\|\nabla u\|_{2}^{2}+c_{2}\left\|\nabla u_{t}\right\|_{2}^{2}+c_{3}(g \circ \nabla u)(t)+\lambda_{3} \Phi_{3}(t)
\end{aligned}
$$


and

$$
\begin{aligned}
L(t) & \geq E(t)-c_{1}\|\nabla u\|_{2}^{2}-c_{2}\left\|\nabla u_{t}\right\|_{2}^{2}-c_{3}(g \circ \nabla u)(t)+\lambda_{3} \Phi_{3}(t) \\
& \geq \frac{1}{\rho+2}\left\|u_{t}\right\|_{\rho+2}^{\rho+2}+\left(\frac{1}{2}(1-l)-c_{1}\right)\|\nabla u(t)\|_{2}^{2} \\
& +\left(\frac{1}{2}-c_{2}\right)\left\|\nabla u_{t}(t)\right\|_{2}^{2}+\left(\frac{1}{2}-c_{3}\right)(g \circ \nabla u)(t) \\
& +\frac{1}{p+2}\|u(t)\|_{p+2}^{p+2}+\lambda_{3} \Phi_{3}(t)
\end{aligned}
$$

where $c_{1}=\frac{\lambda_{1}\left(c_{s}^{2}+\rho+1\right)}{2(\rho+1)}, c_{2}=\frac{\left(\lambda_{1}+\lambda_{2}\right)\left(\alpha_{1}+\rho+1\right)}{2(\rho+1)}$, and $c_{3}=\frac{l\left(\rho+1+c_{s}^{2}\right) \lambda_{2}}{2(\rho+1)}$. Hence, selecting $\lambda_{i}, i=$ 1, 2 such that

$$
\begin{aligned}
& \lambda_{1}<\min \left\{\frac{(\rho+1)(1-l)}{c_{s}^{2}+\rho+1}, \frac{\rho+1}{\alpha_{1}+\rho+1}\right\}, \\
& \lambda_{2}<\min \left\{\frac{\rho+1}{l\left(c_{s}^{2}+\rho+1\right)}, \frac{\rho+1}{\alpha_{1}+\rho+1}-\lambda_{1}\right\},
\end{aligned}
$$

and again from the definition of $E(t)$, there exist two positive constants $\beta_{1}$ and $\beta_{2}$ such that

$$
\beta_{1}\left(E(t)+\Phi_{3}(t)\right) \leq L(t) \leq \beta_{2}\left(E(t)+\Phi_{3}(t)\right), \quad t \geq 0
$$

To obtain a better estimate for $\int_{\Omega} \nabla u \int_{0}^{t} g(t-s) \nabla u(s) \mathrm{d} s \mathrm{~d} x$, we need the following Lemma which repeats Lemma 2 in [25].

Lemma 3.2. For $t \geq 0$, we have

$$
\begin{aligned}
\int_{\Omega} \nabla u \int_{0}^{t} g(t-s) \nabla u(s) \mathrm{d} s \mathrm{~d} x= & \frac{1}{2}\left(\int_{0}^{t} g(s) \mathrm{d} s\right)\|\nabla u\|_{2}^{2}+\frac{1}{2} \int_{0}^{t} g(t-s)\|\nabla u(s)\|_{2}^{2} \mathrm{~d} s \\
& -\frac{1}{2}(g \circ \nabla u)(t) .
\end{aligned}
$$

Proof. Straightforward computations yield this identity.

Now, we are ready to state and prove our result. First, we introduce the following notations as in $[24,25]$. For every measurable set $A \subset R^{+}$, we define the probability measure $\widehat{g}$ by

$$
\widehat{g}(A)=\frac{1}{l} \int_{A} g(s) \mathrm{d} s .
$$

The flatness set and the flatness rate of $g$ are defined by

$$
F_{g}=\left\{s \in R^{+} \mid g(s)>0 \text { and } g^{\prime}(s)=0\right\}
$$

and

$$
R_{g}=\widehat{g}\left(F_{g}\right)=\frac{1}{l} \int_{F_{g}} g(s) \mathrm{d} s .
$$


Before proceeding, we note that there exists $t_{0}>0$ such that

$$
\int_{0}^{t} g(s) \mathrm{d} s \geq \int_{0}^{t_{0}} g(s) \mathrm{d} s=g_{*}>0, \quad \forall t \geq t_{0}
$$

since $g$ is nonnegative and continuous.

Theorem 3.3. Let $\left(u_{0}, u_{1}\right) \in H_{0}^{1}(\Omega) \times H_{0}^{1}(\Omega)$ be given. Suppose that (A1)-(A3), $(2,1)$ and the hypothesis on $p$ hold. Assume further that $R_{g}<\frac{g_{*} \alpha}{l\left(2 \alpha+2 c_{s}^{2}\right)}, H(0)<\frac{g_{*}(4+l)-3 l}{4}$ and $g_{*}>\frac{3 l}{4+l}$ with

$$
\alpha=\frac{\left(g_{*}(4+l)-3 l\right)(1-l)^{p}}{8 c_{s}^{2(p+1)}(2 E(0))^{p}} .
$$

Then the solution energy of (1.4)-(1.6) satisfies

$$
E(t) \leq K \xi(t)^{-\mu}, \quad t \geq 0,
$$

where $\mu$ and $K$ are positive constants.

Proof. In order to obtain the decay result of $E(t)$, it suffices to prove that of $L(t)$. To this end, we need to estimate the derivative of $L(t)$. It follows from (3.2) and Eq. (1.4) that

$$
\begin{aligned}
\Phi_{1}^{\prime}(t) & =\frac{1}{\rho+1}\left\|u_{t}\right\|_{\rho+2}^{\rho+2}+\left\|\nabla u_{t}\right\|_{2}^{2}-\|\nabla u\|_{2}^{2} \\
& +\int_{\Omega} \nabla u \int_{0}^{t} g(t-s) \nabla u(s) \mathrm{d} s \mathrm{~d} x-\|u\|_{p+2}^{p+2}
\end{aligned}
$$

which together with the identity (3.6) and (2.2) gives

$$
\begin{aligned}
\Phi_{1}^{\prime}(t) & \leq \frac{1}{\rho+1}\left\|u_{t}\right\|_{\rho+2}^{\rho+2}+\left\|\nabla u_{t}\right\|_{2}^{2}-\left(1-\frac{l}{2}\right)\|\nabla u\|_{2}^{2} \\
& +\frac{1}{2} \int_{0}^{t} g(t-s)\|\nabla u(s)\|_{2}^{2} \mathrm{~d} s-\frac{1}{2}(g \circ \nabla u)(t)-\|u\|_{p+2}^{p+2} .
\end{aligned}
$$

Next, we would like to estimate $\Phi_{2}^{\prime}(t)$. Taking a derivative of $\Phi_{2}$ in (3.3) and using Eq. (1.4) to get

$$
\begin{aligned}
\Phi_{2}^{\prime}(t) & =\left(1-\int_{0}^{t} g(s) \mathrm{d} s\right) \int_{\Omega} \nabla u(t) \int_{0}^{t} g(t-s)(\nabla u(t)-\nabla u(s)) \mathrm{d} s \mathrm{~d} x \\
& +\int_{\Omega}\left(\int_{0}^{t} g(t-s)(\nabla u(t)-\nabla u(s)) \mathrm{d} s\right)^{2} \mathrm{~d} x-\frac{1}{\rho+1}\left(\int_{0}^{t} g(s) \mathrm{d} s\right)\left\|u_{t}\right\|_{\rho+2}^{\rho+2} \\
& -\left(\int_{0}^{t} g(s) \mathrm{d} s\right)\left\|\nabla u_{t}\right\|_{2}^{2}-\int_{\Omega} \nabla u_{t}(t) \int_{0}^{t} g^{\prime}(t-s)(\nabla u(t)-\nabla u(s)) \mathrm{d} s \mathrm{~d} x \\
& -\frac{1}{\rho+1} \int_{\Omega}\left|u_{t}\right|^{\rho} u_{t} \int_{0}^{t} g^{\prime}(t-s)(u(t)-u(s)) \mathrm{d} s \mathrm{~d} x \\
& +\int_{\Omega}|u|^{p} u \int_{0}^{t} g(t-s)(u(t)-u(s)) \mathrm{d} s \mathrm{~d} x .
\end{aligned}
$$


We now estimate the first two terms on the right-hand side of (3.11) as in [25]. Indeed, for all measure set $A$ and $F$ such that $A=R^{+}-F$, we have

$$
\begin{aligned}
\int_{\Omega} & \nabla u(t) \int_{0}^{t} g(t-s)(\nabla u(t)-\nabla u(s)) \mathrm{d} s \mathrm{~d} x \\
= & \int_{\Omega} \nabla u(t) \int_{A \cap[0, t]} g(t-s)(\nabla u(t)-\nabla u(s)) \mathrm{d} s \mathrm{~d} x \\
& +\int_{\Omega} \nabla u(t) \int_{F \cap[0, t]} g(t-s)(\nabla u(t)-\nabla u(s)) \mathrm{d} s \mathrm{~d} x \\
\leq & \int_{\Omega} \nabla u(t) \int_{A \cap[0, t]} g(t-s)(\nabla u(t)-\nabla u(s)) \mathrm{d} s \mathrm{~d} x \\
& \left.+\int_{F \cap[0, t]} g(s) \mathrm{d} s\right)\|\nabla u\|_{2}^{2}-\int_{\Omega} \nabla u(t) \int_{F \cap[0, t]} g(t-s) \nabla u(s) \mathrm{d} s \mathrm{~d} x .
\end{aligned}
$$

To simplify notations, we denote

$$
A_{t}=A \cap[0, t] \text { and } F_{t}=F \cap[0, t] .
$$

Using Hölder inequality Young's inequality and (2.2), we see that, for $\delta_{1}>0$,

$$
\begin{aligned}
& \int_{\Omega} \nabla u(t) \int_{A_{t}} g(t-s)(\nabla u(t)-\nabla u(s)) \mathrm{d} s \mathrm{~d} x \\
& \quad \leq \delta_{1}\|\nabla u\|_{2}^{2}+\frac{l}{4 \delta_{1}} \int_{\Omega} \int_{A_{t}} g(t-s)|\nabla u(t)-\nabla u(s)|^{2} \mathrm{~d} s \mathrm{~d} x
\end{aligned}
$$

and

$$
\begin{aligned}
& \int_{\Omega} \nabla u(t) \int_{F_{t}} g(t-s) \nabla u(s) \mathrm{d} s \mathrm{~d} x \\
& \leq \frac{1}{2}\left(\int_{F_{t}} g(s) \mathrm{d} s\right)\|\nabla u\|_{2}^{2}+\frac{1}{2} \int_{F_{t}} g(t-s)\|\nabla u(s)\|_{2}^{2} \mathrm{~d} s .
\end{aligned}
$$

Thus, from the definition of $\widehat{g}(F)$ by (3.8), (3.12) becomes

$$
\begin{aligned}
\int_{\Omega} & \nabla u(t) \int_{0}^{t} g(t-s)(\nabla u(t)-\nabla u(s)) \mathrm{d} s \mathrm{~d} x \\
\leq & \left(\delta_{1}+\frac{3}{2} \widehat{\jmath g}(F)\right)\|\nabla u\|_{2}^{2}+\frac{l}{4 \delta_{1}} \int_{\Omega} \int_{A_{t}} g(t-s)|\nabla u(t)-\nabla u(s)|^{2} \mathrm{~d} s \mathrm{~d} x \\
& +\frac{1}{2} \int_{F_{t}} g(t-s)\|\nabla u(s)\|_{2}^{2} \mathrm{~d} s .
\end{aligned}
$$

The second term on the right-hand side of (3.11) can be estimated as follows (see [25]), for $\delta_{2}>0$, 


$$
\begin{aligned}
& \int_{\Omega}\left(\int_{0}^{t} g(t-s)(\nabla u(t)-\nabla u(s)) \mathrm{d} s\right)^{2} \mathrm{~d} x \\
& \quad=\int_{\Omega}\left(\int_{A_{t}} g(t-s)(\nabla u(t)-\nabla u(s)) \mathrm{d} s+\int_{F_{t}} g(t-s)(\nabla u(t)-\nabla u(s)) \mathrm{d} s\right)^{2} \mathrm{~d} x \\
& \leq\left(1+\frac{1}{\delta_{2}}\right) l \int_{\Omega} \int_{A_{t}} g(t-s)|\nabla u(t)-\nabla u(s)|^{2} \mathrm{~d} s \mathrm{~d} x \\
& \quad+\left(1+\delta_{2}\right) \widehat{l g}(F) \int_{\Omega} \int_{F_{t}} g(t-s)|\nabla u(t)-\nabla u(s)|^{2} \mathrm{~d} s \mathrm{~d} x .
\end{aligned}
$$

Using Hölder inequality Young's inequality and (A2) to deal with the fifth term, for $\delta_{3}>0$,

$$
\begin{aligned}
& \left|\int_{\Omega} \nabla u_{t}(t) \int_{0}^{t} g^{\prime}(t-s)(\nabla u(t)-\nabla u(s)) \mathrm{d} s \mathrm{~d} x\right| \\
& \quad \leq \delta_{3}\left\|\nabla u_{t}\right\|_{2}^{2}+\frac{1}{4 \delta_{3}} \int_{\Omega}\left(\int_{0}^{t} g^{\prime}(t-s)(\nabla u(t)-\nabla u(s)) \mathrm{d} s\right)^{2} \mathrm{~d} x \\
& \quad \leq \delta_{3}\left\|\nabla u_{t}\right\|_{2}^{2}-\frac{g(0)}{4 \delta_{3}}\left(g^{\prime} \circ \nabla u\right)(t) .
\end{aligned}
$$

Exploiting Hölder inequality Young's inequality Lemma 2.1 and (A2) to estimate the sixth term, for $\delta_{4}>0$,

$$
\begin{aligned}
& \left.\left|\frac{1}{\rho+1} \int_{\Omega}\right| u_{t}\right|^{\rho} u_{t} \int_{0}^{t} g^{\prime}(t-s)(u(t)-u(s)) \mathrm{d} s \mathrm{~d} x \mid \\
& \quad \leq \frac{1}{\rho+1}\left(\delta_{4}\left\|u_{t}\right\|_{2(\rho+1)}^{2(\rho+1)}+\frac{1}{4 \delta_{4}} \int_{\Omega}\left(\int_{0}^{t} g^{\prime}(t-s)(u(t)-u(s)) \mathrm{d} s\right)^{2} \mathrm{~d} x\right) \\
& \leq \frac{1}{\rho+1}\left(\delta_{4} c_{s}^{2(\rho+1)}\left\|\nabla u_{t}\right\|_{2}^{2(\rho+1)}-\frac{g(0) c_{s}^{2}}{4 \delta_{4}}\left(g^{\prime} \circ \nabla u\right)(t)\right) \\
& \leq \frac{1}{\rho+1}\left(\alpha_{1} \delta_{4}\left\|\nabla u_{t}\right\|_{2}^{2}-\frac{g(0) c_{s}^{2}}{4 \delta_{4}}\left(g^{\prime} \circ \nabla u\right)(t)\right) .
\end{aligned}
$$

For the last term, thanks to Hölder inequality Young's inequality Lemma 2.1, (2.7), (2.2) and (3.8), we have, for $\delta_{5}>0$,

$$
\begin{aligned}
& \left.\left|\int_{\Omega}\right| u\right|^{p} u \int_{0}^{t} g(t-s)(u(t)-u(s)) \mathrm{d} s \mathrm{~d} x \mid \\
& \leq \delta_{5} \int_{\Omega}|u|^{2(p+1)} \mathrm{d} x+\frac{1}{4 \delta_{5}} \int_{\Omega}\left(\int_{0}^{t} g(t-s)(u(t)-u(s)) \mathrm{d} s\right)^{2} \mathrm{~d} x \\
& \leq \delta_{5} \alpha_{2}\|\nabla u\|_{2}^{2}+\frac{l c_{s}^{2}}{2 \delta_{5}} \int_{\Omega} \int_{A_{t}} g(t-s)|\nabla u(t)-\nabla u(s)|^{2} \mathrm{~d} s \mathrm{~d} x \\
& +\frac{l c_{s}^{2}}{2 \delta_{5}} \widehat{g}(F) \int_{\Omega} \int_{F_{t}} g(t-s)|\nabla u(t)-\nabla u(s)|^{2} \mathrm{~d} s \mathrm{~d} x,
\end{aligned}
$$


where $\alpha_{2}=c_{s}^{2(p+1)}\left(\frac{2 E(0)}{1-l}\right)^{p}$. Thus, gathering these estimates (3.13)-(3.17) and using (3.9), we obtain, for $t \geq t_{0}$,

$$
\begin{aligned}
\Phi_{2}^{\prime}(t) & \leq\left(\left(1-g_{*}\right)\left(\delta_{1}+\frac{3}{2} \sqrt{g}(F)\right)+\delta_{5} \alpha_{2}\right)\|\nabla u\|_{2}^{2} \\
& -\left(\frac{g(0)}{4 \delta_{3}}+\frac{g(0) c_{s}}{4(\rho+1) \delta_{4}}\right)\left(g^{\prime} \circ \nabla u\right)(t)+\frac{1-g_{*}}{2} \int_{F_{t}} g(t-s)\|\nabla u(s)\|_{2}^{2} \mathrm{~d} s \\
& +\left(\delta_{3}+\frac{\alpha_{1} \delta_{4}}{\rho+1}-g_{*}\right)\left\|\nabla u_{t}\right\|_{2}^{2}-\frac{g_{*}}{(\rho+1)}\left\|u_{t}\right\|_{\rho+2}^{\rho+2} \\
& +l\left(1+\frac{1}{\delta_{2}}+\frac{c_{s}^{2}}{2 \delta_{5}}+\frac{1-g_{*}}{4 \delta_{1}}\right) \int_{\Omega} \int_{A_{t}} g(t-s)|\nabla u(t)-\nabla u(s)|^{2} \mathrm{~d} s \mathrm{~d} x \\
& +\left(1+\delta_{2}+\frac{c_{s}^{2}}{2 \delta_{5}}\right) \widehat{\nwarrow}(F) \iint_{\Omega} g(t-s)|\nabla u(t)-\nabla u(s)|^{2} \mathrm{~d} s \mathrm{~d} x .
\end{aligned}
$$

Further, taking a derivative of $\Phi_{3}(t)$, using the fact that $\frac{\xi^{\prime}(t)}{\xi(t)}=\eta(t)$ is a decreasing function and the definition of $\Phi_{3}(t)$ by (3.4), we derive that (see [25])

$$
\begin{aligned}
\Phi^{\prime}{ }_{3}(t) & =H(0)\|\nabla u\|_{2}^{2}-\int_{0}^{t} \frac{\xi^{\prime}(t-s)}{\xi(t-s)} H(t-s)\|\nabla u(s)\|_{2}^{2} \mathrm{~d} s-\int_{0}^{t} g(t-s)\|\nabla u(s)\|_{2}^{2} \mathrm{~d} s \\
& \leq H(0)\|\nabla u\|_{2}^{2}-\eta(t) \int_{0}^{t} H(t-s)\|\nabla u(s)\|_{2}^{2} \mathrm{~d} s-\int_{0}^{t} g(t-s)\|\nabla u(s)\|_{2}^{2} \mathrm{~d} s \\
& =H(0)\|\nabla u\|_{2}^{2}-\eta(t) \Phi_{3}(t)-\int_{0}^{t} g(t-s)\|\nabla u(s)\|_{2}^{2} \mathrm{~d} s .
\end{aligned}
$$

Hence, we conclude from (2.6), (3.10), (3.18) and (3.19) that for any $t \geq t_{0}>0$,

$$
\begin{aligned}
L^{\prime}(t)= & E^{\prime}(t)+\lambda_{1} \Phi_{1}^{\prime}(t)+\lambda_{2} \Phi_{2}^{\prime}(t)+\lambda_{3} \Phi_{3}^{\prime}(t) \\
\leq & \left.\frac{1}{2}-\lambda_{2}\left(\frac{g(0)}{4 \delta_{3}}+\frac{g(0) c_{s}^{2}}{4(\rho+1) \delta_{4}}\right)\right)\left(g^{\prime} \circ \nabla u\right)(t) \\
& +\frac{1}{\rho+1}\left(\lambda_{1}-\lambda_{2} g_{*}\right)\left\|u_{t}\right\|_{\rho+2}^{+\rho 2}-\lambda_{1}\|u\|_{p+2}^{p+2}-\frac{\lambda_{1}}{2}(g \circ \nabla u)(t) \\
& +\left(\lambda_{1}+\lambda_{2}\left(\delta_{3}+\frac{\alpha_{1} \delta_{4}}{\rho+1}-g_{*}\right)\right)\left\|\nabla u_{t}\right\|_{2}^{2}+\left(\frac{\lambda_{1}}{2}-\lambda_{3}\right) \int_{0}^{t} g(t-s)\|\nabla u(s)\|_{2}^{2} \mathrm{~d} s \\
& +\left(\lambda_{2}\left(1-g_{*}\right)\left(\delta_{1}+\frac{3}{2}\lceil\widehat{g}(F))+\lambda_{2} \delta_{5} \alpha_{2}-\lambda_{1}\left(1-\frac{l}{2}\right)+\lambda_{3} H(0)\right)\|\nabla u\|_{2}^{2}\right. \\
& +\lambda_{2} l\left(1+\frac{1}{\delta_{2}}+\frac{c_{s}^{2}}{2 \delta_{5}}+\frac{1-g_{*}}{4 \delta_{1}}\right) \int_{\Omega} \int_{A_{t}} g(t-s)|\nabla u(t)-\nabla u(s)|^{2} \mathrm{~d} s \mathrm{~d} x \\
& +\lambda_{2}\left(1+\delta_{2}+\frac{c_{s}^{2}}{2 \delta_{5}}\right)\left\lceil\widehat{g}(F) \int_{\Omega} \int_{F_{t}} g(t-s)|\nabla u(t)-\nabla u(s)|^{2} \mathrm{~d} s \mathrm{~d} x\right. \\
& +\lambda_{2}\left(\frac{1-g_{*}}{2}\right) \int_{F_{t}} g(t-s)\|\nabla u(s)\|_{2}^{2} \mathrm{~d} s-\lambda_{3} \eta(t) \Phi_{3}(t) .
\end{aligned}
$$

For $n \in \mathcal{N}$, we consider the sets (see $[24,25])$

$$
A_{n}=\left\{s \in R^{+} \mid n g^{\prime}(s)+g(s) \leq 0\right\}
$$


and observe that

$$
\bigcup_{n} A_{n}=R^{+}-\left\{F_{g} \cup N_{g}\right\}
$$

where $F_{g}$ is given in (3.7) and $N_{g}$ is the null set where $g^{\prime}$ is not defined. In addition, denoting $F_{n}=R^{+}-A_{n}$, then

$$
\lim _{n \rightarrow \infty} \widehat{g}\left(F_{n}\right)=\widehat{g}\left(F_{g}\right)
$$

because $A_{n}$ are increasingly nested. Thus, choosing $A=A_{n}, F=F_{n}$ and $\lambda_{1}=\left(g_{*}-\varepsilon\right)$ $\lambda_{2}$ for some $\varepsilon>0$ in (3.20), we obtain

$$
\begin{aligned}
L^{\prime}(t) & \leq\left(\frac{1}{2}-\lambda_{2}\left(\frac{g(0)}{4 \delta_{3}}+\frac{g(0) c_{s}^{2}}{4(\rho+1) \delta_{4}}\right)\right)\left(g^{\prime} \circ \nabla u\right)(t) \\
& -\frac{\lambda_{2} \varepsilon}{\rho+1}\left\|u_{t}\right\|_{\rho+2}^{\rho+2}+\lambda_{2}\left(\delta_{3}+\frac{\alpha_{1} \delta_{4}}{\rho+1}-\varepsilon\right)\left\|\nabla u_{t}\right\|_{2}^{2}-\left(g_{*}-\varepsilon\right) \lambda_{2}\|u\|_{p+2}^{p+2} \\
& -\frac{\left(g_{*}-\varepsilon\right) \lambda_{2}}{2}(g \circ \nabla u)(t)+\left(\frac{\left(g_{*}-\varepsilon\right) \lambda_{2}}{2}-\lambda_{3}\right) \int_{0}^{t} g(t-s)\|\nabla u(s)\|_{2}^{2} \mathrm{~d} s \\
& +\left(\lambda_{2}\left(1-g_{*}\right)\left(\delta_{1}+\frac{3}{2}\left\lceil\widehat{g}\left(F_{n}\right)\right)+\lambda_{2} \delta_{5} \alpha_{2}-\lambda_{2}\left(g_{*}-\varepsilon\right)\left(1-\frac{l}{2}\right)+\lambda_{3} H(0)\right)\|\nabla u\|_{2}^{2}\right. \\
& +\lambda_{2} l\left(1+\frac{1}{\delta_{2}}+\frac{c_{s}^{2}}{2 \delta_{5}}+\frac{1-g_{*}}{4 \delta_{1}}\right) \int_{\Omega} \int_{A_{n t}} g(t-s)|\nabla u(t)-\nabla u(s)|^{2} \mathrm{~d} s \mathrm{~d} x \\
& +\lambda_{2}\left(1+\delta_{2}+\frac{c_{s}^{2}}{2 \delta_{5}}\right)\left\lceil\widehat{g}\left(F_{n}\right) \int_{\Omega} \int_{F_{n t}} g(t-s)|\nabla u(t)-\nabla u(s)|^{2} \mathrm{~d} s \mathrm{~d} x\right. \\
& +\lambda_{2}\left(\frac{1-g_{*}}{2}\right) \int_{F_{n t}} g(t-s)\|\nabla u(s)\|_{2}^{2} \mathrm{~d} s-\lambda_{3} \eta(t) \Phi_{3}(t) .
\end{aligned}
$$

At this point, we take $\delta_{3}=\delta_{4}<\frac{(\rho+1) \varepsilon}{2\left(\rho+1+\alpha_{1}\right)}$ and select $\lambda_{2}$ so that

$$
\frac{1}{2}-\lambda_{2}\left(\frac{g(0)}{4 \delta_{3}}+\frac{g(0) c_{s}^{2}}{4(\rho+1) \delta_{4}}\right) \geq \frac{1}{4}
$$

then (3.21) becomes

$$
\begin{aligned}
L^{\prime}(t) & \leq-\frac{\lambda_{2} \varepsilon}{\rho+1}\left\|u_{t}\right\|_{\rho+2}^{\rho+2}-\frac{\lambda_{2} \varepsilon}{2}\left\|\nabla u_{t}\right\|_{2}^{2}-\left(g_{*}-\varepsilon\right) \lambda_{2}\|u\|_{p+2}^{p+2} \\
& -\frac{\left(g_{*}-\varepsilon\right) \lambda_{2}}{2}(g \circ \nabla u)(t)+\left(\frac{\left(g_{*}-\varepsilon\right) \lambda_{2}}{2}-\lambda_{3}\right) \int_{0}^{t} g(t-s)\|\nabla u(s)\|_{2}^{2} \mathrm{~d} s \\
& +\left(\lambda_{2}\left(1-g_{*}\right)\left(\delta_{1}+\frac{3}{2} \widehat{\jmath}\left(F_{n}\right)\right)+\lambda_{2} \delta_{5} \alpha_{2}-\lambda_{2}\left(g_{*}-\varepsilon\right)\left(1-\frac{l}{2}\right)+\lambda_{3} H(0)\right)\|\nabla u\|_{2}^{2} \\
& +\left(\lambda_{2} l\left(1+\frac{1}{\delta_{2}}+\frac{c_{s}^{2}}{2 \delta_{5}}+\frac{1-g_{*}}{4 \delta_{1}}\right)-\frac{1}{4 n}\right) \int_{\Omega} \int_{A_{n t}} g(t-s)|\nabla u(t)-\nabla u(s)|^{2} \mathrm{~d} s \mathrm{~d} x \\
& +\lambda_{2}\left(1+\delta_{2}+\frac{c_{s}^{2}}{2 \delta_{5}}\right) \widehat{\zeta g}\left(F_{n}\right) \iint_{\Omega} g(t-s)|\nabla u(t)-\nabla u(s)|^{2} \mathrm{~d} s \mathrm{~d} x \\
& +\lambda_{2}\left(\frac{1-g_{*}}{2}\right) \int_{F_{n t}} g(t-s)\|\nabla u(s)\|_{2}^{2} \mathrm{~d} s-\lambda_{3} \eta(t) \Phi_{3}(t) .
\end{aligned}
$$


For $\varepsilon, \delta_{2}$ small enough and large value of $n$ and $t_{0}$, we see that if

$$
\delta_{5}=\alpha \text { and } \widehat{g}\left(F_{n}\right)<\frac{g_{*} \alpha}{l\left(2 \alpha+2 c_{s}^{2}\right)}
$$

then

$$
\left(\left(1+\delta_{2}\right)+\frac{c_{s}^{2}}{2 \delta_{5}}\right) \widehat{l g}\left(F_{n}\right)-\frac{g_{*}-\varepsilon}{2} \leq 0
$$

and

$$
\frac{3}{2}\left(1-g_{*}\right) \widehat{l}\left(F_{n}\right)<\delta\left(g_{*}-\varepsilon\right)\left(1-\frac{l}{2}\right),
$$

where

$$
\alpha=\frac{g_{*}(4+l)-3 l}{8 \alpha_{2}}=\frac{\left(g_{*}(4+l)-3 l\right)(1-l)^{p}}{8 c_{s}^{2(p+1)}(2 E(0))^{p}}
$$

and

$$
\delta=\frac{3\left(1-g_{*}\right) l}{g_{*}(4-2 l)} .
$$

Note that $\alpha>0$ and $0<\delta<1$ due to $g_{*}>\frac{3 l}{4+l}$. Furthermore, we require $\lambda_{2}$ and $\lambda_{3}$ satisfying

$$
\lambda_{2} l\left(1+\frac{1}{\delta_{2}}+\frac{c_{s}^{2}}{2 \delta_{5}}+\frac{1-g_{*}}{4 \delta_{1}}\right)-\frac{1}{4 n}<0
$$

and

$$
\frac{\lambda_{2}}{2}<\lambda_{3}<\frac{\lambda_{2}\left(g_{*}(4+l)-3 l\right)}{8 H(0)},
$$

this is possible because of $H(0)<\frac{g_{*}(4+l)-3 l}{4}$. Then, letting $\delta_{1}$ be small enough and using (3.22), we see that

$$
\lambda_{2}\left(1-g_{*}\right)\left(\delta_{1}+\frac{3}{2} / \widehat{g}\left(F_{n}\right)\right)+\lambda_{2} \delta_{5} \alpha_{2}-\lambda_{2}\left(g_{*}-\varepsilon\right)\left(1-\frac{l}{2}\right)+\lambda_{3} H(0) \leq 0 .
$$

Hence, from the definition of $E(t)$ by (2.4), we have, for all $t \geq t_{0}$,

$$
L^{\prime}(t) \leq-c_{4} E(t)-\lambda_{3} \eta(t) \Phi_{3}(t)
$$

for some positive constant $c_{4}$. As $\eta(t)$ is decreasing, we have $\eta(t) \leq c_{4}$ after some $t_{*} \geq$ $t_{0}$. Hence, with the help of the right hand side inequality in (3.5), we find

$$
L^{\prime}(t) \leq-c_{5} \eta(t) L(t), \quad \forall t \geq t_{*}
$$

for some positive constant $c_{5}>0$. An integration of (3.23) over $\left(t_{*}, t\right)$ gives

$$
L(t) \leq L\left(t_{*}\right) \mathrm{e}^{-c_{5} \int_{t_{*}}^{t} \eta(s) \mathrm{d} s}, \quad \forall t \geq t_{*} .
$$


Then using the left hand side inequality in (3.5) leads to

$$
\beta_{1}\left(E(t)+\Phi_{3}(t)\right) \leq L\left(t_{*}\right) \mathrm{e}^{-c_{5} \int_{t_{*}}^{t} \eta(s) \mathrm{d} s}, \quad \forall t \geq t_{*}
$$

Therefore, by virtue of the continuity and boundedness of $E(t)$ and $\xi(t)$ on the interval $\left[0, t_{*}\right]$, we infer that

$$
E(t) \leq K \xi(t)^{-\mu}, t \geq 0,
$$

for some positive constants $K$ and $\mu$.

Similar to those remarks as in [25], we have the following remark.

Remark. Note that there is a wide class of relaxation functions satisfying (A3). More precisely, if $\xi(t)=\mathrm{e}^{\alpha t}, \alpha>0$, then $\eta(t)=\alpha$, this gives the exponential decay estimate $E(t) \leq c_{1} \mathrm{e}^{-c_{2} \alpha t}$, for some positive constants $c_{1}$ and $c_{2}$. Similarly, if $\xi(t)=(1+t)^{\alpha}, \alpha>$ 0 , then we obtain the polynomial decay estimate $E(t) \leq c_{3}(1+t)^{-\mu}$, for some positive constants $c_{3}$ and $\mu$.

\section{Acknowledgements}

The authors would like to thank very much the anonymous referees for their valuable comments on this work.

\section{Competing interests}

The author declares that they have no competing interests.

Received: 16 February 2011 Accepted: 6 October 2011 Published: 6 October 2011

\section{References}

1. Berrimi, S, Messaoudi, SA: Existence and decay of solutions of a viscoelastic equation with a nonlinear source. Nonlinear Anal Theory Methods Appl. 64, 2314-2331 (2006). doi:10.1016/j.na.2005.08.015

2. Berrimi, S, Messaoudi, SA: Exponential decay of solutions to a viscoelastic equation with nonlinear localized damping. Electron J Diff Equ. 88, 1-10 (2004)

3. Cavalcanti, MM, Domingos Cavalcanti, VN, Ferreira, J: Existence and uniform decay of nonlinear viscoelastic equation with strong damping. Math Methods Appl Sci. 24, 1043-1053 (2001). doi:10.1002/mma.250

4. Cavalcanti, MM, Domingos Cavalcanti, VN, Soriano, JA: Exponential decay for the solution of semilinear viscoelastic wave equation with localized damping. Electron J Diff Equ. 44, 1-14 (2002)

5. Cavalcanti, MM, Domingos Cavalcanti, VN, Prates Filho, JS, Soriano, JA: Existence and uniform decay rates for viscoelastic problems with nonlinear boundary damping. Diff Integr Equ. 14(1), 85-116 (2001)

6. Cavalcanti, MM, Oquendo, HP: Frictional versus viscoelastic damping in a semilinear wave equation. SIAM J Contro Optim. 42(4), 1310-1324 (2003). doi:10.1137/S0363012902408010

7. Fabrizo, M, Polidoro, S: Asymptotic decay for some differential systems with fading memory. Appl Anal. 81, 1245-1264 (2002). doi:10.1080/0003681021000035588

8. Furati, K, Tatar, N-e: Uniform boundedness and stability for a viscoelastic problem. Appl Math Comput. 167, 1211-1220 (2005). doi:10.1016/j.amc.2004.08.036

9. Han, X, Wang, M: Global existence and uniform decay for a nonlinear viscoelastic equation with damping. Nonlinear Anal Theory Methods Appl. 70, 3090-3098 (2009). doi:10.1016/j.na.2008.04.011

10. Kawashima, S, Shibata, Y: Global existence and exponential stability of small solutions to nonlinear viscoelasticity. Commun Math Phys. 148, 189-208 (1992). doi:10.1007/BF02102372

11. Kirane, M, Tatar, N-e: A memory type boundary stabilization of a mildy damped wave equation. Electron J Qual Theory Diff Equ. 6, 1-7 (1999)

12. Liu, WJ: General decay rate estimate for a viscoelastic equation with weakly nonlinear time-dependent dissipation and source terms. J Math Phys. 50, 113506 (2009). doi:10.1063/1.3254323

13. Medjden, $\mathrm{M}$, Tatar, $\mathrm{N}$-e: Asymptotic behavior for a viscoelastic problem with not necessarily decreasing kernel. Appl Math Comput. 167, 1221-1235 (2005). doi:10.1016/j.amc.2004.08.035

14. Messaoudi, SA, Tatar, N-e: Exponential and polynomial decay for quasilinear viscoelastic equation. Nonlinear Anal Theory Methods Appl. 68, 785-793 (2007)

15. Messaoudi, SA, Tatar, N-e: Global existence and asymptotic behavior for a nonlinear viscoelastic problem. Math Sci Res J. 7(4), 136-149 (2003)

16. Messaoudi, SA, Tatar, N-e: Global existence and uniform stability of solutions for a quasilinear viscoelastic problem. Math Methods Appl Sci. 30, 665-680 (2007). doi:10.1002/mma.804

17. Messaoudi, SA: Blow-up and global existence in a nonlinear viscoelastic wave equation. Math Nachr. 260, 58-66 (2003). doi:10.1002/mana.200310104

18. Messaoudi, SA: Blow-up of positive-initial-energy solutions of a nonlinear viscoelastic hyperbolic equation. J Math Anal Appl. 320, 902-915 (2006). doi:10.1016/j.jmaa.2005.07.022

19. Messaoudi, SA: General decay of the solution energy in a viscoelastic equation with a nonlinear source. Nonlinear Anal Theory Methods Appl. 69, 2589-2598 (2008). doi:10.1016/j.na.2007.08.035 
20. Messaoudi, SA: General decay of solutions of a viscoelastic equation. J Math Anal Appl. 341, 1457-1467 (2008). doi:10.1016/j.jmaa.2007.11.048

21. Messaoudi, SA, Tatar, N-e: Exponential decay for a quasilinear viscoelastic equation. Math Nachr. 282, 1443-1450 (2009). doi:10.1002/mana.200610800

22. Munoz Rivera, JE, Lapa, EC, Baretto, R: Decay rates for viscoelastic plates with memory. J Elast. 44, 61-87 (1996). doi:10.1007/BF00042192

23. Nečas, MJ, Šverák, V: On weak solutions to a viscoelasticity model. Comment Math Univ Carolin. 31(3), 557-565 (1990)

24. Pata, V: Exponential stability in linear viscoelasticity. Q Appl Math. 64, 499-513 (2006)

25. Tatar, N-e: Arbitrary decay in linear viscoelasticity. J Math Phys. 52, 013502 (2010)

26. Wu, ST: Blow-up of solutions for an integro-differential equation with a nonlinear source. Electron J Diff Equ. 45, 1-9 (2006)

27. Wu, ST: General decay of solutions for a viscoelastic equation with nonlinear damping and source terms. Acta Math Sci. 31(4), 1436-1448 (2011)

28. Wu, ST: General decay of energy for a viscoelastic equation with linear damping and source term. Taiwan J Math. (in press)

29. Hrusa, WJ: Global existence and asymptotic stability for a nonlinear hyperbolic Volterra equation with large initial data. SIAM J Math Anal. 16, 110-134 (1985). doi:10.1137/0516007

30. Medjden, M, Tatar, N-e: On the wave equation with a temporal nonlocal term. Dyn Syst Appl. 16, 665-672 (2007)

31. Tiehu, Q: Asymptotic behavior of a class of abstract integrodifferential equations and applications. J Math Anal Appl. 233, 130-147 (1999). doi:10.1006/jmaa.1999.6271

doi:10.1186/1687-2770-2011-28

Cite this article as: Wu: Arbitrary decays for a viscoelastic equation. Boundary Value Problems 2011 2011:28.

\section{Submit your manuscript to a SpringerOpen ${ }^{\circ}$} journal and benefit from:

- Convenient online submission

- Rigorous peer review

- Immediate publication on acceptance

- Open access: articles freely available online

- High visibility within the field

- Retaining the copyright to your article

Submit your next manuscript at $\boldsymbol{s p r i n g e r o p e n . c o m ~}$ 